\title{
Radiological changes in left lobe amoebic liver abscesses
}

\author{
S. RAMACHANDRAN \\ M.D., M.R.C.P., M.R.C.P.E. \\ Colombo North General Hospital, Ragama, Sri Lanka
}

\begin{abstract}
Summary
Elevation of the left dome of the diaphragm occurred in $43 \%$ of cases with left lobe amoebic liver abscesses. It was rarely seen in patients with an abscess in the right hepatic lobe. Elevation of the right dome of the diaphragm, which occurred in $36 \%$ of cases, is also compatible with a diagnosis of left lobe abscess. This radiological sign was present in $75 \%$ of cases with right lobe abscesses. Pleuro-pulmonary changes in the left chest, when associated with an elevation of the left dome of the diaphragm, favours the diagnosis of an amoebic abscess of the left lobe of the liver. Radiological changes in barium studies are usually confined to patients with large hepatic abscesses. In clinical practice the information obtained from simple radiology can provide adequate diagnostic confirmation of the presence of a left lobe liver abscess.
\end{abstract}

\section{Introduction}

By virtue of its anatomical position, an amoebic abscess arising in the left lobe of the liver frequently presents as a mass in the epigastrium (Paul, 1960). Extension of the abscess into the pericardial cavity (Wilmot, 1962) and the frequency of intraperitoneal rupture (Alkan, Kalmi and Kalderon, 1961) stress the need for early diagnosis of a left lobe abscess even before the formation of an epigastric mass. In order to define the value of simple radiology in the diagnosis of a left lobe liver abscess, the radiological changes in patients with amoebic liver abscesses have been studied with reference to the anatomical site.

\section{Patients and methods}

Posteroanterior teleradiograms of the chest were taken in seventy-four cases of amoebic liver abscess. Pus was demonstrated in all the cases either by closed aspiration or at laparotomy. Of the seventy-four cases, fourteen had an abscess situated in the left lobe of the liver. The relevant clinical features in these fourteen cases were correlated with the radiological abnormalities observed. Plain X-rays of the abdomen and barium studies were done in some cases with left lobe abscesses.

\section{Results}

Radiological changes observed in cases with left lobe abscesses

Six patients $(43 \%)$ had an elevation of the left dome of the diaphragm. In two of the cases the right and left domes were at the same level, while in the remaining four cases the left dome was at a level up to 1.6 in $(4.2 \mathrm{~cm})$ above the right (Figs. 1 and 2 ; Table 1). Elevation of the right dome of the diaphragm was observed in five cases (36\%). In these cases the right dome was significantly higher than the left by $1.2-1.6$ in $(3.1-4.2 \mathrm{~cm})$, Fig. 3 .

No change in the relationship between the domes of the diaphragm was present in three of the cases

TABLE 1. The incidences of the radiological changes in left lobe abscesses, right lobe abscesses and all cases of hepatic abscess

\begin{tabular}{lccc}
\hline Radiological change & $\begin{array}{c}\text { Left lobe } \\
\text { abscess, } \\
\text { fourteen cases }\end{array}$ & $\begin{array}{c}\text { Right lobe } \\
\text { abscess, } \\
\text { sixty cases }\end{array}$ & $\begin{array}{c}\text { All cases of } \\
\text { abscesses, } \\
\text { seventy-four cases }\end{array}$ \\
\hline Elevation of left dome & 6 & 1 & 7 \\
Elevation of right dome & $(43 \%)$ & $(1.8 \%)$ & $(9 \%)$ \\
No change in diaphragm & 5 & 45 & 50 \\
Left pleuro-pulmonary reactions & $(36 \%)$ & $(75 \%)$ & $(68 \%)$ \\
Right pleuro-pulmonary reactions & 3 & 14 & 17 \\
& $(21 \%)$ & $(23 \%)$ & $(23 \%)$ \\
& 2 & 1 & 3 \\
& $(14 \%)$ & $(1.8 \%)$ & $(4 \%)$ \\
& nil & 19 & 19 \\
\end{tabular}




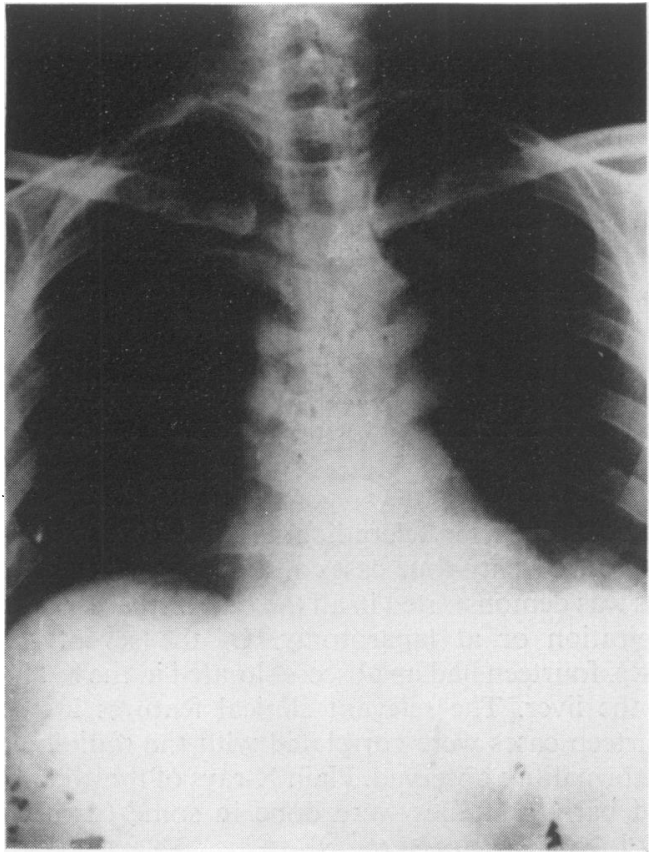

FIG. 1. There is an elevation of the left dome of the diaphragm, and hence the right and the left domes are at the same level. A left basal pleural reaction is present.

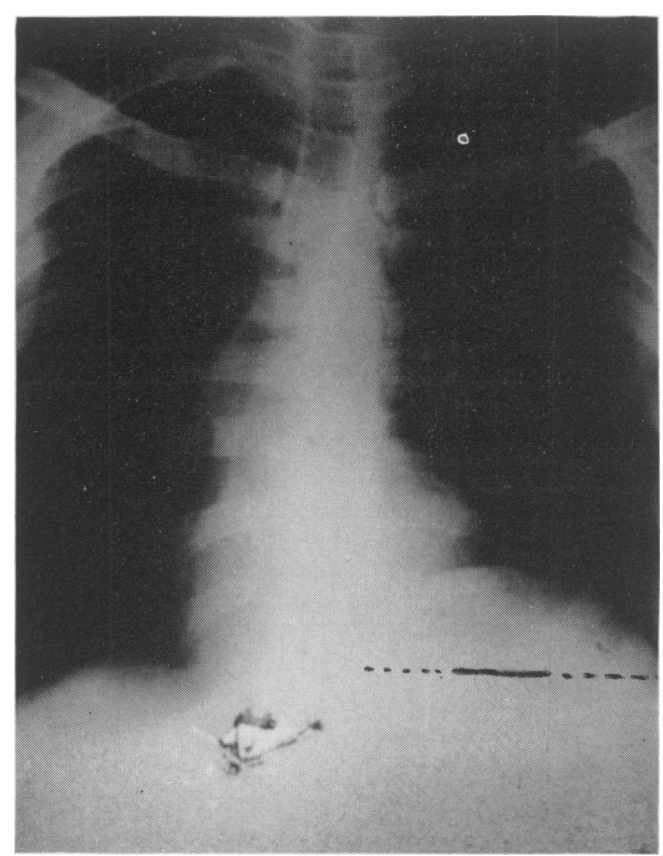

Fig. 2. Shows marked elevation of the left hemidiaphragm. The left dome was 1.6 in above the right.

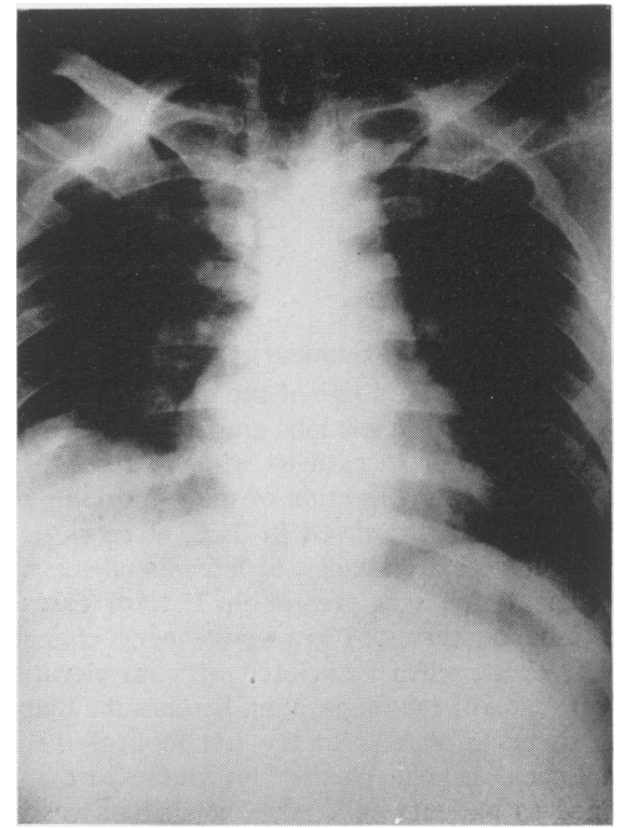

Fig. 3. Significant elevation of the right dome of the diaphragm in a case of left lobe liver abscess.

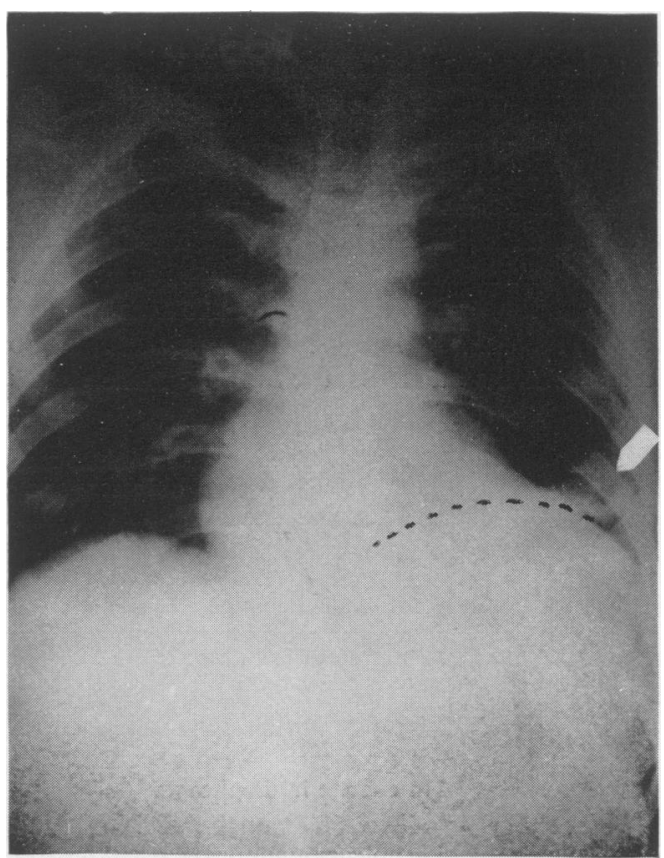

FIg. 4. There is a left basal lung abscess with an elevation of the left dome of the diaphragm. This patient had a left lobe amoebic abscess with downward hepatomegaly. 


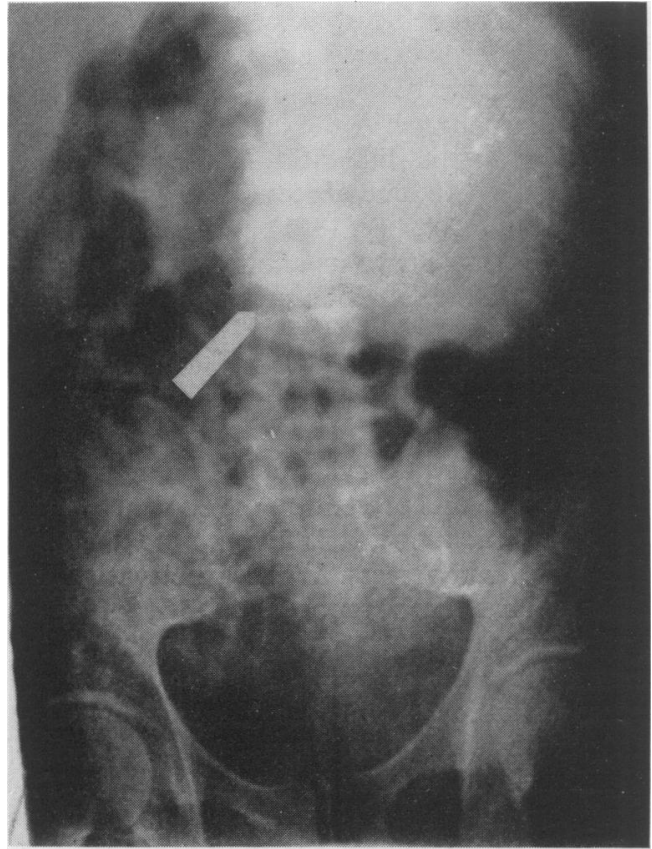

FIG. 5. A large soft tissue mass occupies the left epigastric region extending into the left hypochondrium. A case of left lobe abscess with a palpable epigastric mass.

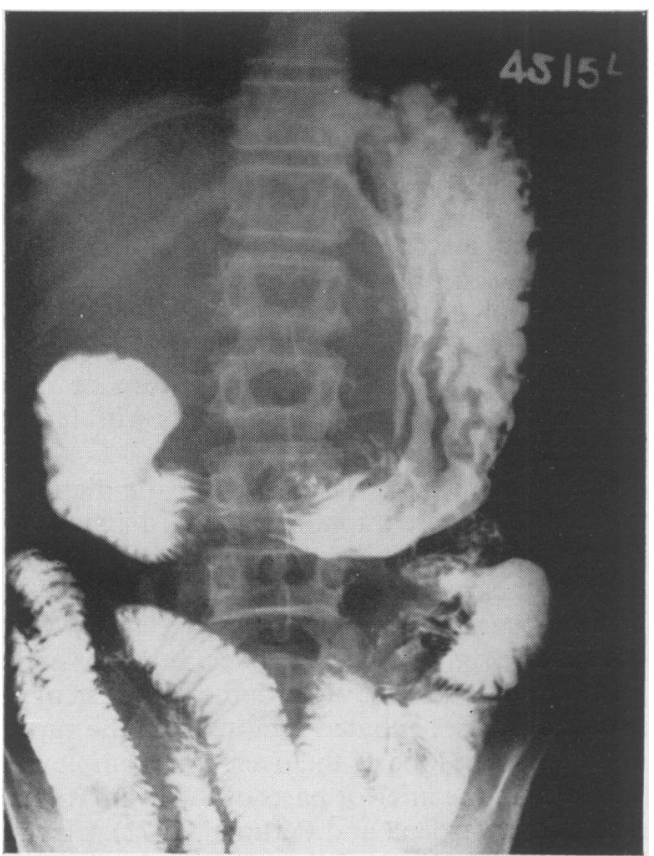

FIG. 6. Barium meal showing the stomach and the first part of the duodenum stretched around a soft tissue mass. The soft tissue mass appears to be continuous with the liver.

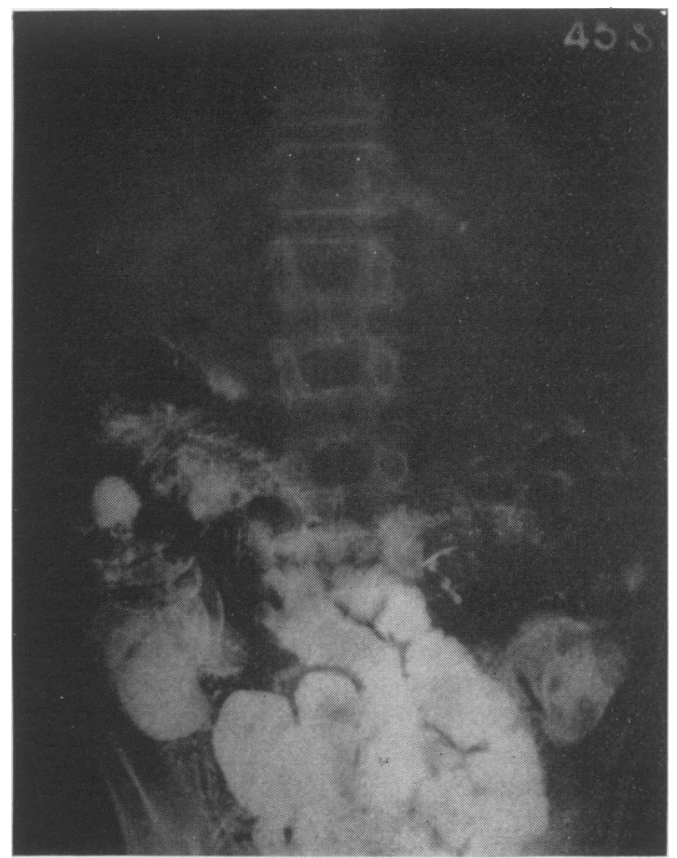

Fig. 7. Barium meal showing the transverse colon displaced downward by a soft tissue mass continuous with the liver.

$(21 \%)$. Pleuropulmonary changes were seen in the left chest in two cases $(14 \%)$. In one of them, there was a small basal lung abscess while in the other there was a basal pleural reaction (Figs. 1 and 4). Both cases had an associated elevation of the left dome of the diaphragm. Pleuropulmonary reactions were not present in the right chest in any of the cases.

Plain X-ray of the abdomen in four of the cases showed a soft tissue mass in the epigastrium (Fig. 5), while a barium meal in two of the above four cases showed the stomach and the first part of the duodenum stretched around the soft tissue mass. There was also a downward displacement of the transverse colon (Figs. 6 and 7).

Relationship between the radiological changes and the clinical features in cases of left lobe abscesses

In seven patients there was a localized mass in the epigastrium. Three cases had a palpable liver mainly a left lobe while a single case had a diffuse hepatomegaly. The remaining three cases had no downward hepatic enlargement.

Five of the six cases with radiological evidence of an elevation of the left dome of the diaphragm had either a localized epigastric mass or downward hepatic enlargement. A single case with an elevation of the left dome of the diaphragm, had a nonpalpable liver. Similarly, three of the five cases with 
an elevation of the right dome of the diaphragm had either a localized mass or a palpable liver. The remaining two cases had no downward hepatomegaly. Of the two cases with no abnormalities in the levels of the domes of the diaphragm, one had an enlarged liver mainly of the left lobe while the other had a mass in the epigastrium. The patients with soft tissue masses and abnormalities in the barium meal X-rays, as would have been expected, had a localized epigastric mass. The radiological abnormalities in the chest X-rays bore no distinct relationship to the physical signs in this group.

\section{Radiological changes observed in cases with right lobe abscesses}

Elevation of the right dome of the diaphragm was present in forty-five of the sixty cases $(75 \%)$. On the other hand, an elevation of the left dome was observed in only one case $(1.8 \%)$. No abnormality in the levels of the diaphragm occurred in fourteen of the cases $(23 \%)$. Pleuropulmonary changes were present in twenty cases $(33 \%)$. In all but one of the cases the changes were in the lower zones of the right chest (Fig. 8; Table 1).

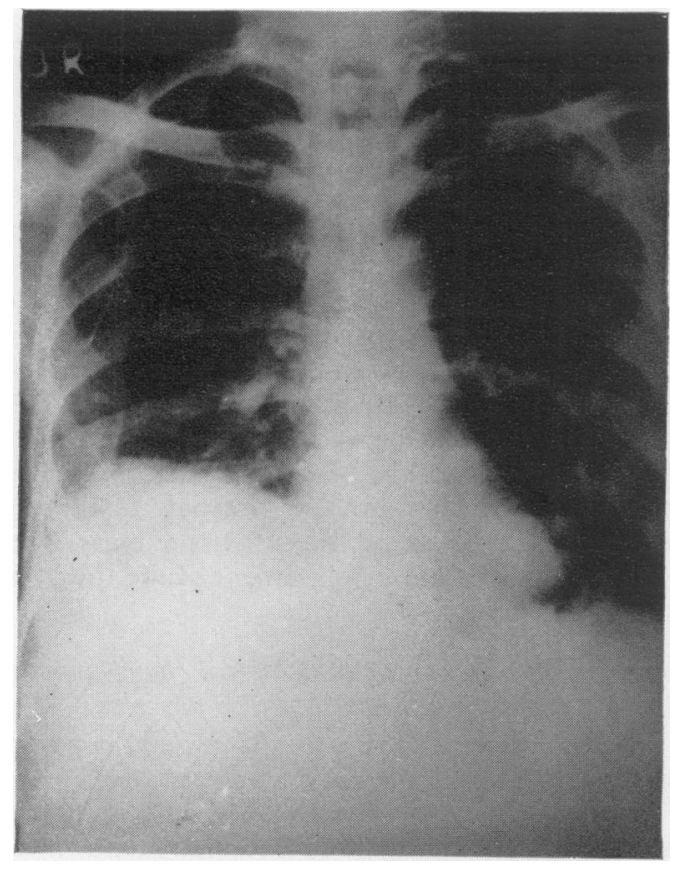

FIG. 8. There is a left basal lung abscess with a fluid level in a patient with an amoebic abscess in the right lobe of the liver. There is an elevation of the right dome of the diaphragm.

\section{Discussion}

Although the radiological changes occurring in patients with amoebic abscesses of the right lobe of the liver have been extensively studied, comparatively little attention has been paid to the changes occurring in abscesses situated in the left lobe. Reference has been drawn to the difficulties in demonstrating the presence of a left lobe abscess by simple radiology (DeBakey and Ochsner, 1951), while it has also been reported that, owing to its anatomical relationships, an abscess of the left lobe may not become radiologically evident until it is about to rupture into the thoracic cage (Lamont and Pooler, 1958).

Observations from this study, however, show that in the absence of distention of the stomach or intestines, an elevation of the left dome of the diaphragm in patients presenting the clinical manifestations of hepatic amoebiasis favours the diagnosis of a left lobe liver abscess. While an elevation of the left dome has been reported in $5 \%$ of cases of hepatic amoebiasis, no reference was made to the presence or absence of pus or to the site of the abscess in patients with pus (Lamont and Pooler, 1958 ); in this study it was nearly always confined to the patients with left lobe hepatic abscesses. Two of the eight patients with proven left lobe abscesses showed this radiological abnormality (Alkan et al., 1961 ), and it was observed in $43 \%$ of the patients in this study. By occurring in patients both with and without an epigastric mass or palpable liver, an elevation of the left dome indicated that it may be ac valuable radiological sign in the early diagnosis of:an abscess of the left hepatic lobe. The left lobe of the liver is closely related to the medial third of the left diaphragm and an elevation of the left dome suggests hepatic enlargement horizontally towards the left and superiorly towards the thorax. In this respect it may also be a valuable radiological sign of an impending rupture into the thoracic cage. Downward extension of the enlarging abscess cavity, owing to the abdominal cavity offering less resistance to expansion, would account for the absence of left dome elevation in $57 \%$ of the patients with left lobe abscesses.

An elevation of the right dome of the diaphragm appears also to be compatible with a diagnosis of a left hepatic abscess. This radiological sign was present in $36 \%$ of the cases, a striking figure when compared with its occurrence in $75 \%$ of the cases with abscesses in the right lobe of the liver. It is thus evident than an elevation of the right dome could occur with amoebic abscesses situated remote from the superior pole of the right lobe of the liver, for example in the left lobe or in the inferior parts of the liver (Ramachandran, Jayawardena and Perumal, 1971). Elevation of the right dome of the diaphragm in patients with a left lobe abscess would suggest either an associated non-specific hepatic reaction, 'amoebic hepatitis' (Ramachandran, Sivalingam and Perumal, 1973), 
the presence of multiple abscesses (Paul, 1960), or a generalized hepatic congestion (Lamont and Pooler, 1958).

Although pleuropulmonary reactions in the left chest occurred in abscesses situated either in the right or left lobes of the liver, left pleuropulmonary reactions in association with an elevation of the left dome of the diaphragm once again favour a diagnosis of an amoebic abscess of the left lobe.

The presence of soft tissue shadows in the plain $X$-rays of the abdomen and abnormalities in the barium studies were only present with the large abscesses with palpable left hepatic lobes or in the presence of an epigastric mass. In this respect these radiological signs, while being useful confirmatory evidence, have their limitations in the diagnosis of smaller abscesses arising in the left lobe of the liver. Enlargement of the cardiac shadow due to fluid in the pericardial sac, although not observed in this study, could be a valuable radiological sign in the diagnosis of a left lobe abscess (Wilmot, 1962).

It thus appears that in clinical practice the information obtained from simple radiology could, in a proportion of patients, provide an adequate diagnostic confirmation of the presence of a left lobe liver abscess. Its value is even more evident as the incidence of hepatic amoebiasis is often highest in regions with a rapid turnover of patients where sophisticated radio-diagnostic procedures are not readily available.

\section{References}

Alkan, W.J., Kalmi, B. \& Kalderon, M. (1961) The clinical syndrome of amoebic abscesses of the left lobe of the liver. Annals of Internal Medicine, 55, 801.

DABAKeY, M.E. \& OChSNeR, A. (1951) Hepatic amoebiasis. International Abstracts of Surgery, 92, 209.

LAmont, McE. N. \& Pooler, N.R. (1958) Hepatic amoebiasis. Quarterly Journal of Medicine, 27, 389.

Paul, M. (1960) New concepts in amoebic abscess of the liver. The British Journal of Surgery, 47, 502.

Ramachandran, S., Jayawardena, D.L.N. \& Perumal, J.R.A. (1971) Radiological changes in hepatic amoebiasis. Postgraduate Medical Journal, 47, 615.

Ramachandran, S., Sivalingam, S. \& Perumal, J.R.A. (1973) Concepts in hepatic amoebiasis. Journal of Tropical Medicine and Hygiene, 76, 39.

Wilmot, A.J. (1962) Clinical Amoebiasis. Blackwell Scientific Publications: Oxford. 